\title{
Diversidade de bromélias epífitas na Reserva Particular do Patrimônio Natural Serra do Teimoso - Jussari, BA
}

\author{
Joice Rodrigues de Mendonça Reis ${ }^{1}$ \& Talita Fontoura ${ }^{2,3}$ \\ ${ }^{1}$ Programa de Pós-Graduação em Desenvolvimento Regional e Meio Ambiente, \\ Universidade Estadual de Santa Cruz - UESC, \\ Rod. Ilhéus-Itabuna, Km 16, CEP 45650-000, Ilhéus, BA, Brasil,e-mail: joice@cabruca.org.br \\ ${ }^{2}$ Departamento de Ciências Biológicas, Universidade Estadual de Santa Cruz-UESC, \\ Rodovia Ilhéus-Itabuna, Km 16, CEP 45650-000, Ilhéus, BA, Brasil \\ ${ }^{3}$ Autor para correspondência: Talita Fontoura, e-mail: talita_fontoura@uol.com.br
}

REIS, J.R. de M. \& FONTOURA, T. 2009. Diversity of epiphytic bromeliads in the Reserva Particular do Patrimônio Natural Serra do Teimoso - Jussari, BA. Biota Neotrop. 9(1): http://www.biotaneotropica.org.br/ v9n1/en/abstract?article+bn01209012009.

\begin{abstract}
Vascular epiphytes are frequent in mesic habitats and mid-elevation regions. The present study investigated the diversity and species composition of epiphytic bromeliads in the Natural Reserve of Serra do Teimoso (RNST) located in a transitional area between ombrophilous and semideciduous forests. Adapted from the "Rapid and Representative Sampling of Vascular and Non-vascular epiphyte Diversity of Tropical Rain Forests" protocol, our survey method used eight phorophytes of Cariniana legalis (Martius) Kuntze found between 284 and $573 \mathrm{~m}$ a.s.l. We registered 19 morphospecies and 526 bromeliad groups. Almost one third of the species were classified as widely distributed and $27.3 \%$ are endemic to southern Bahia. Shannon index was 2.2 nats.ind $^{-1}$ and the estimated number of species in this area was $25(\mathrm{SD}= \pm 3.5)$. Most $C$. legalis harbored a similar abundance and species composition of epiphytes, which was dominated by Hohenbergia and Aechmea species. This study registered the occurrence of three species that are new to the state of Bahia, and one genus was collected for the first time in the RNST. Results are in accordance with the known pattern of lower epiphytic diversity in drier locations. Since all new records are exclusive to the crowns of large trees, the sampling of these new records was only possible because climbing techniques were used. Compared to other methodologies for floristic surveys, the one employed here demanded lower sample effort and yielded similar results. Large trees play an important role for epiphytes due to the concentration of species and individuals on them. Thus, the utilization of canopy methodologies in other field surveys would be desirable to sample appropriately epiphytes in large trees.

Keywords: conservation, semideciduous forest, epiphytes, Atlantic Rain Forest, Bromeliaceae.
\end{abstract}

REIS, J.R. de M. \& FONTOURA, T. 2009. Diversidade de bromélias epífitas na Reserva Particular do Patrimônio Natural Serra do Teimoso - Jussari, BA. Biota Neotrop. 9(1): http://www.biotaneotropica.org.br/ v9n1/pt/abstract?article+bn01209012009.

Resumo: A presença de epífitas vasculares em florestas tropicais está freqüentemente associada a ambientes mésicos e de altitude média. Este trabalho objetivou investigar a diversidade e a composição de espécies de bromélias epífitas na Reserva Natural da Serra do Teimoso que é uma área de transição entre as florestas ombrófila e a semidecídua. A amostragem foi adaptada do "Protocolo para Amostragem Rápida e Representativa da Diversidade de Epífitas Vasculares e Não-Vasculares de Florestas Tropicais" utilizando-se oito indivíduos de Cariniana legalis localizados entre $285 \mathrm{~m}$ e $573 \mathrm{~m}$ de altitude. Foram registradas 19 morfoespécies e 526 grupos de bromélias. Quase um terço das espécies possui distribuição ampla e 27,3\% é endêmica do sul da Bahia. O índice de Shannon foi 2,2 nats.indivíduo ${ }^{-1}$ e o número de espécies estimado foi $25(\mathrm{SD}= \pm 3,5)$. A maioria dos indivíduos de Jequitibá possuiu abundância e composição de bromélias semelhantes entre si com predominância de espécies de Aechmea e Hohenbergia. Três novas ocorrências para o sul da Bahia e mais um gênero ainda não coletado na área foram registrados. Os resultados estão de acordo com o padrão de menor diversidade de epífitas em florestas mais secas. Somente com técnicas específicas para acessar as grandes árvores do dossel, foi possível registrar as novas ocorrências, pois tais espécies são exclusivas das copas das grandes árvores. Comparada as outras metodologias para levantamentos florísticos, a metodologia empregada teve um baixo esforço amostral apresentando resultados semelhantes. As grandes árvores possuem especial importância para as epífitas devido à concentração de espécies e de indivíduos sobre elas. Assim, seria aconselhável que outros levantamentos utilizassem metodologias de acesso ao dossel para coletar epífitas mais apropriadamente nas grandes árvores.

Palavras-chave: conservação, floresta semi-decídua, epífitas, Floresta Atlântica, Bromeliaceae. 


\section{Introdução}

A presença de epífitas vasculares em florestas tropicais está freqüientemente associada a ambientes mésicos e de altitude média. Geralmente, a riqueza é maior nas altitudes intermediárias das montanhas, havendo certa variabilidade de acordo com os locais inventariados. Por exemplo, trabalhos desenvolvidos em escala local no México, Colômbia e Equador, apontam respectivamente, picos de riqueza de espécies a $1.430 \mathrm{~m}, 2.350-2.600 \mathrm{~m}$ e a $1.600 \mathrm{~m}$ de altitude (Krömer et al. 2005). Por outro lado, plantas epífitas são infreqüentes em ambientes que possuem baixa umidade ou que passem por algum período de seca (Gentry \& Dodson 1987, Benzing 1990). Segundo Gentry \& Dodson (1987) o epifitismo é representado nestes locais, por espécies das famílias mais adaptadas ao epifitismo como cactáceas, bromeliáceas e orquidáceas.

A Floresta Atlântica na região sul da Bahia possui suas áreas variando desde locais úmidos próximos ao litoral até áreas mais secas, que são substituídas pela caatinga na região a oeste do estado. Gouvêa et al. (1976) classificaram estas formações florestais como mata higrófila (na porção a leste), mata mesófila e matas de cipó (nas regiões a oeste). Em algumas regiões, a transição entre matas higrófilas e mesófilas pode ocorrer entre 80-100 km da costa, como é o caso da região de Floresta Atlântica localizada em Jussari que possui componentes florísticos de florestas mais secas (Jardim 2003, Amorim et al. 2005). Embora a composição florística da região de Jussari seja relativamente conhecida (Amorim et al. 2005), os únicos estudos referentes às epífitas são provenientes de Una (Alves 2005) que se localiza em linha reta a aproximadamente $80 \mathrm{~km}$ à leste de Jussari, na floresta higrófila.

Nas matas higrófilas da Bahia, a maioria das bromélias epífitas pertence à subfamília Bromelioideae e os estudos conduzidos até o momento sugerem que amostrar espécies de bromélia com esta forma de vida significa amostrar a maioria das espécies que possam ocorrer em áreas de mata higrófila (Alves 2005). Estas epífitas ocorrem com menos frequiência em árvores com diâmetros pequenos (ca. de $5 \mathrm{~cm}$ ) e mais freqüentemente em árvores que possuem diâmetros acima de $10 \mathrm{~cm}$. A maior freqüência de epífitas em árvores com grandes diâmetros aparentemente não é uma exclusividade das bromélias das matas higrófilas sul baianas, mas ocorre de maneira geral em outras áreas de florestas tropicais (Migenis \& Ackerman 1993, Zotz \& Vollrath 2003). Além deste padrão de ocupação das árvores, levantamentos realizados nas florestas do Panamá (Nieder et al. 2001), México, Bolívia e Venezuela têm indicado que a área necessária para amostrar a maioria das espécies epífitas a ocorrer em uma região é relativamente pequena se comparada à área necessária para amostrar a maioria das espécies de plantas terrestres (Nieder et al. 1999, Gradstein et al. 2003). Estas características da comunidade epífita levaram Gradstein et al. (2003) a desenvolverem um protocolo rápido de levantamento da diversidade de epífitas juntamente com a utilização de estimadores de riqueza (Colwell \& Codington 1994, Gotelli \& Colwell 2001). Tais protocolos rápidos e a utilização de estimadores representam uma ferramenta a mais para auxiliar na tomada de decisões de cunho conservacionista, sendo desejáveis, por exemplo, para a região sul da Bahia que possui cerca de $47 \%$ de sua área ocupada por pastagens e agricultura e $14 \%$ por florestas em estágio inicial de regeneração (Landau 2003).

Assim, este trabalho objetiva determinar a diversidade de espécies de bromélias epífitas em uma área de transição entre as matas higrófila e mesófila e discutir aspectos sobre a conservação e a ocupação do hábitat de epífitas.

\section{Material e Métodos}

\section{1. Área de estudo}

O estudo foi realizado na Reserva Particular do Patrimônio Natural da Serra do Teimoso (RNST; $15^{\circ} 08^{\prime} \mathrm{S}$ e $39^{\circ} 31^{\prime} \mathrm{W}$ ), município de Jussari, Bahia (Figura 1). As partes mais altas da RNST atingem 850 m.s.m. e a vegetação pode ser classificada como transicional entre as florestas ombrófila densa e a estacional semidecidual (Veloso 1991). A vegetação é permanentemente úmida nos topos de morro e semidecídua somente nas altitudes mais baixas (Jardim 2003). A temperatura média anual é de $23,5^{\circ} \mathrm{C}$, a precipitação anual varia de 1.200 a $1.600 \mathrm{~mm}$ anuais, havendo 2 a 3 meses mais secos entre junho e agosto. $\mathrm{O}$ clima é Am na classificação de Köppen (Landau 2003).

A RNST possui ca. de 200 ha e possui um dossel bastante descontínuo, com árvores de grande porte que podem atingir $50 \mathrm{~m}$ de altura e até $35 \mathrm{~cm}$ de diâmetro. Amorim et al. (2005) assinalam que várias espécies vegetais de grande valor econômico estão presentes nesta área: pau-brasil (Caesalpinia echinata Lam.); jequitibá (Cariniana legalis (Martius) Kuntze); oiticica (Brosimum guianense (Aubl.) Huber); sapucaia (Lecythis pisonis (Cambess.) Miers); pau-d'arco (Tabebuia bilbergii (Bureau \& K.Schum.) Standl.), dentre outras.

Dados sobre as espécies vegetais coletadas estão disponíveis em http://www.nybg.org/bsci/hcol/netr/ e os trabalhos sobre o dossel da RNST estão disponíveis em http://www.ib.unicamp.br/profs/fsantos/ ne313/index.html

\section{Amostragem}

A coleta de dados foi feita durante uma semana do mês de abril de 2005. A metodologia de amostragem de oito árvores foi adaptada do "Protocolo para Amostragem Rápida e Representativa da Diversidade de Epífitas Vasculares e Não-Vasculares de Florestas Tropicais" (Gradstein et al. 2003). Este protocolo recomenda a amostragem em oito árvores adultas de dossel em 1 ha de floresta, bem como arvoretas e arbustos ao redor de cada árvore, perfazendo uma área de 20 × $20 \mathrm{~m}$ ao redor da árvore selecionada.

Foram desenvolvidos trabalhos piloto, que mostraram a presença de epífitas somente nas copas das árvores com circunferência à altura do peito $(\mathrm{CAP}) \geq 100 \mathrm{~cm}$ e a ausência de epífitas nos troncos das árvores menores (http://www.ib.unicamp.br/profs/fsantos/ne313/ index. html). Cariniana legalis (jequitibá) foi escolhida como espécie foco por ser uma espécie de dossel, ser dominante na área e por possuir muitas epífitas. Uma vez que alguns dos indivíduos encontravam-se separados em linha reta por mais de $100 \mathrm{~m}$, optou-se pela padronização do número de forófitos em detrimento da área de 1 ha recomendada por Gradstein et al. (2003). Assim, oito indivíduos de C. legalis foram escolhidos aleatoriamente no terreno, entre 285 e 573 m.s.m. A altitude e localização de cada um dos forófitos foi obtida através de GPS. Todos os forófitos amostrados possuíam mais de $30 \mathrm{~m}$ de altura. A área de $20 \times 20 \mathrm{~m}$ ao redor dos oito forófitos confirmou a inexistência de epífitas em outras árvores e arbustos ao seu redor.

Os oito forófitos foram escalados através de técnicas de ascensão vertical ("single rope technique", Perry 1978) que são adaptações de técnicas de escalada em rocha às árvores (Padget \& Smith 1989). Diferentemente da técnica empregada por Perry (1978), em todas as ascensões foram utilizadas cordas de segurança.

A abundância de cada uma das espécies foi registrada a partir do centro da copa, contando-se o número de grupos em todos os ramos dos jequitibás. Os grupos foram definidos como qualquer agregado de rosetas de bromélias, fossem eles formados por uma ou por várias rosetas. Grupos distintos foram registrados no caso de haver uma separação visível entre os grupos, sem a emissão de estolões entre um grupo e outro. $\mathrm{O}$ registro dos grupos foi feito 


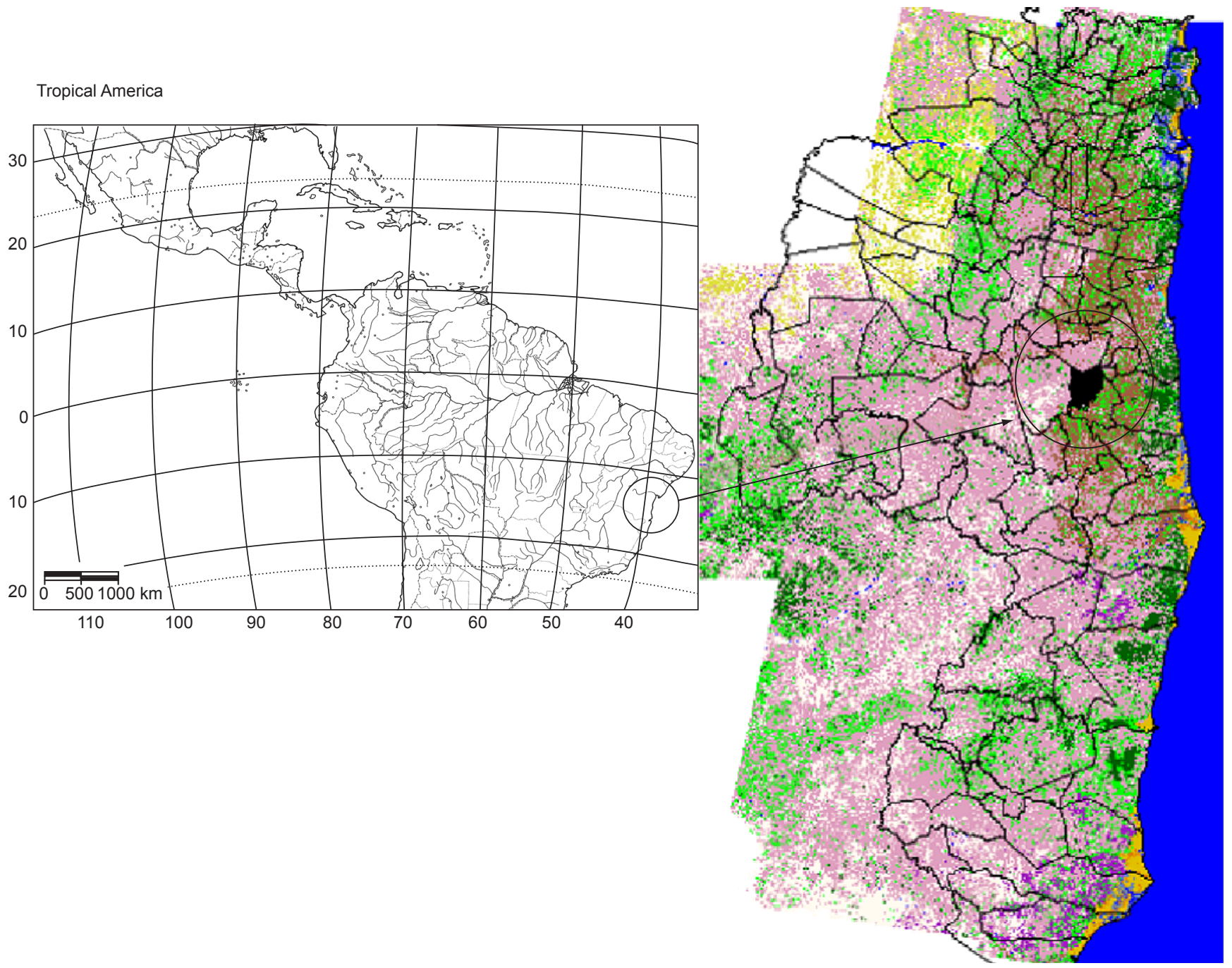

Figura 1. Mapa da área de estudos da região sul da Bahia.

Figure 1. Map of study area located in southern Bahia region.

com o auxílio de binóculos, os grupos foram fotografados para auxiliar na determinação das espécies ou dos morfotipos e quando possível, houve a coleta de material botânico. Além disso, caracteres vegetativos e/ou reprodutivos foram anotados para todos os grupos para também auxiliar na determinação das espécies. Embora Tillandsia usneoides (L.) L. ocorra sobre os forófitos, ela não foi incluída nas análises por necessitar de outro método para a medição de sua abundância.

Tanto o material botânico quanto as fotos foram utilizadas para a elaboração da lista de espécies. Assim, indivíduos estéreis morfologicamente diferentes de todas as demais espécies coletadas foram morfotipados. As exsicatas foram depositadas no herbário da Universidade Estadual de Santa Cruz (HUESC).

A distribuição geográfica das espécies foi determinada através da consulta à bibliografia especializada (Smith \& Downs 1977, 1979, Leme 1987, 1997, 1999, Siqueira Filho \& Leme 2006) e aos herbários do Centro de Pesquisas e Estudos do Cacau (CEPEC) e da Universidade Estadual de Santa Cruz (HUESC). O exame das exsicatas e a consulta à bibliografia permitiram que as espécies fossem agrupadas em: i) ocorrentes do sul da Bahia até o Espírito Santo; ii) ocorrentes na Floresta Atlântica, em outros biomas brasileiros e em outros países; iii) ocorrendo da Floresta Atlântica do sul da Bahia até as regiões ao sul deste bioma; e iv) ocorrentes na Floresta Atlântica da região sudeste até a região nordeste deste bioma.

\section{Análise de dados}

A diversidade de espécies na área e em cada um dos forófitos foi calculada com o índice de Shannon: $H^{\prime}=-\Sigma\left[\left(n_{i} / N\right) \ln \left(n_{i} / \mathrm{N}\right)\right]$ onde: $\mathrm{n}_{\mathrm{i}}=$ número de indivíduos da espécie $\mathrm{i} ; \mathrm{N}=$ número total de indivíduos da amostra.

A estimativa do número total de espécies presente nas árvores foi calculada pelo estimador Chao 2, com correção para sub-amostras, utilizando-se o programa EstimateS (Colwell 1997). As amostras foram aleatorizadas 500 vezes.

\section{Resultados}

Foram registradas 19 morfoespécies e 526 grupos de bromélias sobre jequitibás (Tabela 1). Do total de morfoespécies, $11(57,9 \%)$ foram determinadas até espécie. Três das onze espécies $(27,3 \%)$ 
Tabela 1. Espécies de bromélias epífitas, abundância sobre os forófitos e distribuição geográfica das espécies de Bromeliaceae sobre $C$. legalis na RPPN Serra do Teimoso (Jussari, Bahia). Endêmica - espécie ocorrente do sul da Bahia até o Espírito Santo; Ampla - ocorrentes na Floresta Atlântica, em outros biomas brasileiros e em outros países; FAS - espécie ocorrente da Floresta Atlântica do sul da Bahia até regiões ao sul deste bioma; FASeNe - espécie ocorrente na Floresta Atlântica da região sudeste até a região nordeste.

Table 1. Species, abundance and geographic distribution of epiphytic bromeliads in C. legalis in RPPN Serra do Teimoso (Jussari, Bahia). Endêmica - occurring from south Bahia to state of Espírito Santo; Ampla - occurring in Atlantic Rainforest, other Brazilian biomes and other countries; FAS - occurring from Atlantic Rainforest of south Bahia to southern regions of Atlantic Rainforest; FASeNe - occurring from southeastern Atlantic Rainforest to northeastern Atlantic Rainforest.

\begin{tabular}{|c|c|c|}
\hline Espécies & Abundância de grupos & Distribuição geográfica \\
\hline Aechmea cf. blanchetiana (Baker) L. B. Smith & 4 & Endêmica \\
\hline Aechmea patentissima (Mart. Ex Schult. \& Schult.f.) Baker & 128 & FASeNe \\
\hline Aechmea nudicaulis (L.) Griseb. & 8 & Ampla \\
\hline Aechmea $\mathrm{sp} 1$ & 16 & - \\
\hline Aechmea sp2 & 2 & - \\
\hline Bromeliaceae indet. & 1 & - \\
\hline Edmundoa lindenii (Regel) Leme & 3 & FAS \\
\hline Hohenbergia disjuncta L. B. Sm. & 108 & Endêmica \\
\hline Neoregelia sp1 & 3 & - \\
\hline Neoregelia $\mathrm{sp} 2$ & 5 & - \\
\hline Tillandsia gardnerii Lindl. & 50 & Ampla \\
\hline Tillandsia $\mathrm{sp} 2$ & 3 & - \\
\hline Tillandsia $\mathrm{sp} 1$ & 19 & - \\
\hline Tillandsia tenuifolia $\mathrm{L}$. & 20 & Ampla \\
\hline Vriesea dictyographa Leme & 23 & Endêmica \\
\hline Vriesea flammea L. B. Sm. & 25 & FAS \\
\hline Vriesea ensiformis (Vell.) Beer & 14 & FAS \\
\hline Vriesea paratiensis E.Pereira & 91 & FAS \\
\hline Vriesea $\mathrm{sp} 1$ & 3 & - \\
\hline Total & 526 & - \\
\hline
\end{tabular}

apresentaram distribuição ampla, quatro espécies ocorrem na Floresta Atlântica da região sul do Brasil (36,4\%), três espécies (27,3\%) são endêmicas e uma espécie ocorre na Floresta Atlântica desde a região sudeste até a região nordeste. Edmundoa lindenii, Vriesea flammea e V. paratiensis são novas ocorrências para a região nordeste da Floresta Atlântica, possuindo distribuição anteriormente registrada somente para a região sul da Floresta Atlântica.

No forófito localizado na menor altitude foram encontradas seis espécies de bromélia e no forófito de maior altitude, nove espécies, havendo cinco a dez espécies nos forófitos localizados em altitudes intermediárias (Tabela 2). O mesmo padrão de variação foi observado para a abundância e para a diversidade de bromélias nas árvores das diferentes altitudes. A diversidade geral da área foi de 2,2 nats.indivíduo ${ }^{-1}$. O número total de espécies estimado para a área foi de 25 ( $\mathrm{SD}= \pm 3,5$; Figura 2$)$.

\section{Discussão}

Pouco mais da metade das espécies de Jussari foi determinada, indicando a necessidade de levantamentos florísticos que possibilitem a descrição e a elaboração de chaves de identificação das espécies ocorrentes na região. Jussari representa uma entre outras localidades do sul da Bahia que possuem matas de encosta pouco conhecidas, pois todos os trabalhos que abordam a florística das espécies do sul da Bahia são fortemente centrados em matas de baixada (Smith \& Downs 1974, 1977, 1979, Leme 1997, 1999, Alves 2005). A julgar pela alta diversidade florística do sul da Bahia (Martini et al. 2007), pelo número de espécies publicadas para a família Bromeliaceae na
Tabela 2. Forófitos, altitudes, número de espécies sobre os forófitos (spp), abundância e diversidade (H') de Bromeliaceae epífitas na RPPN Serra do Teimoso (Jussari, Bahia).

Table 2. Phorophytes, altitude, species number in phorophytes (spp), abundance and diversity ( $\mathrm{H}^{\prime}$ ) of epiphytic bromeliads in RPPN Serra do Teimoso (Jussari, Bahia).

\begin{tabular}{ccrrr}
\hline Forófito & Altitude $(\mathbf{m})$ & Spp & Abundância & H' \\
\hline J8 & 573 & 9 & 83 & 1,8 \\
J7 & 479 & 10 & 109 & 1,7 \\
J6 & 439 & 7 & 18 & 1,7 \\
J5 & 439 & 6 & 14 & 1,5 \\
J4 & 429 & 5 & 52 & 1,2 \\
J3 & 421 & 9 & 46 & 2,0 \\
J2 & 295 & 9 & 151 & 1,2 \\
J1 & 285 & 6 & 53 & 1,5 \\
Total & - & 19 & 526 & 2,2 \\
\hline
\end{tabular}

Flora Neotropica (95 espécies, T. Fontoura \& T.Wendt, dados não publicados) e pela alta diversidade em altitudes médias (Cardelús et al. 2006), é provável que algumas das espécies não determinadas de Jussari sejam novas espécies a serem descritas.

O registro de 19 espécies de bromélias sobre uma só espécie arbórea dominante na área (0,89 indivíduos/ha; W. Thomas et al., dados não publicados) e a ausência de epífitas nas árvores de pequeno e médio porte indicam que os grandes jequitibás devem ter 


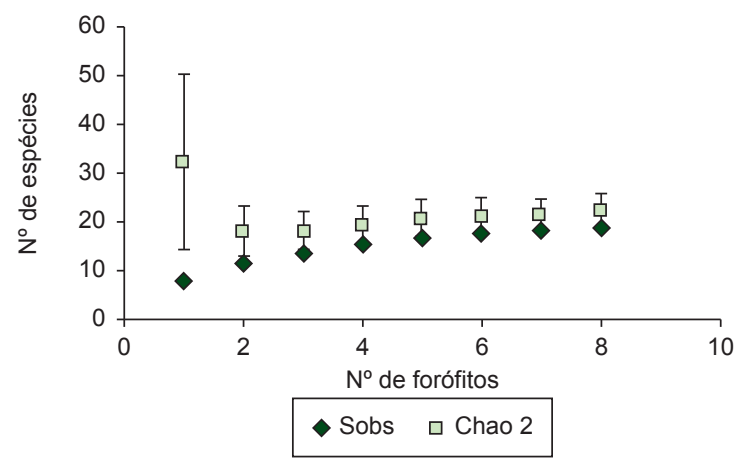

Figura 2. Curva de acumulação de espécies de bromélias epífitas sobre C. legalis na RPPN da Serra do Teimoso (Jussari) Bahia. $\mathrm{S}_{\text {obs }}=$ número de espécies observado; desvios referem-se aos desvios-padrão de cada valor estimado.

Figure 2. Species accumulation curves of epiphytic bromeliads in C. legalis, RPPN Serra do Teimoso (Jussari) Bahia. $\mathrm{S}_{\mathrm{obs}}=$ observed species number; standard deviations represented to each estimated value.

um importante papel para a manutenção da diversidade e riqueza de espécies epífitas em outras áreas de floresta da região.

Trabalhos realizados em escala regional poderão verificar a hipótese de $C$. legalis ser uma espécie também importante para epífitas ao longo da Floresta Atlântica, hospedando esta forma de vida em maior abundância ou riqueza se comparada a outras espécies arbóreas. Esta hipótese é baseada em trabalhos realizados em escala local, que mostraram que algumas espécies arbóreas são desproporcionalmente propícias ao epifitismo (Waechter 1980, Benzing 1990), que C. legalis ocorre desde o estado de Pernambuco até o estado de São Paulo, sendo uma espécie característica do dossel, com grandes diâmetros (Sebben et al. 2000). Uma vez que a Floresta Atlântica é um bioma predominantemente úmido e que árvores com grandes diâmetros são, em sua maioria, forófitos de epífitas (Migenis \& Ackerman 1993, Zotz \& Vollrath 2003, Alves 2005) é provável que esta hipótese seja verdadeira.

Os dados sobre a riqueza e abundância de bromélias por forófito podem ser usados para extrapolar quantas espécies e grupos de bromélias epífitas seriam eliminados do terreno no caso da eliminação de áreas florestais nesta região. Jussari é uma amostra da condição da paisagem a oeste da Floresta Atlântica no sul da Bahia, onde as áreas florestais são reduzidas a manchas de floresta (neste caso, 200 ha de floresta) rodeadas por áreas de pasto (Landau 2003). Deste modo, aproximando a densidade de $C$. legalis encontrada por W. Thomas et al. (dados não publicados) para um indivíduo por hectare, a retirada seletiva de jequitibás em outras matas de encosta de baixa altitude, implicaria na perda de seis a dez espécies e de 46 a 151 grupos de bromélias por hectare. Embora outros levantamentos em fragmentos de mata na região de Jussari sejam necessários, é provável que esta estimativa não esteja longe dos valores reais. Trabalhos desenvolvidos em outras florestas tropicais têm indicado que plantas epífitas possuem uma rápida estabilização da curva de acumulação de espécies devido à acumulação de muitas epífitas em alguns forófitos (Nieder et al. 1999, 2001, Gradstein et al. 2003). Esta característica da comunidade epífita faz com que o número de epífitas a ocorrer em uma região seja rapidamente alcançado utilizando-se uma área amostral relativamente pequena, se comparada à área necessária para amostrar espécies arbóreas (Nieder et al. 1999, 2001).

Comparando-se o número de espécies coletadas e o esforço amostral deste trabalho com o levantamento realizado por Amorim et al. (2005) pode-se notar uma semelhança no número de espécies coletadas e uma grande diferença no esforço amostral. Enquanto o presente trabalho registrou as 19 espécies em uma semana, Amorim et al. (2005) utilizaram os dados provenientes de coletas realizadas entre 1997 e 2004, de coletas acumuladas no Herbário do Centro de Pesquisas do Cacau e de dados de um inventário fitossociológico (W. Thomas et al., dados não publicados). Embora diferenças nas metodologias impeçam comparações em relação às espécies coletadas, a diferença no esforço amostral entre os levantamentos e a semelhança no número de espécies indica que ajustes metodológicos em estudos florísticos e de diversidade seriam altamente desejáveis. Assim, seria interessante que levantamentos florísticos incluíssem a coleta no dossel utilizando diferentes espécies arbóreas de grande porte para amostrar a riqueza e diversidade de bromélias ou de epífitas em geral com um menor esforço amostral. A metodologia de campo aqui adotada permitiu a coleta de um gênero não registrado na área (Neoregelia spp.) pelo levantamento de Amorim et al. (2005) e a coleta de três novas ocorrências para a região nordeste da Floresta Atlântica (Edmundoa lindenii, Vriesea flammea e V.paratiensis). Estas espécies possuíam distribuição geográfica registrada anteriormente somente para a região sul da Floresta Atlântica.

Mais de um quarto $(27,3 \%)$ das bromélias de Jussari tem ocorrência restrita ao eixo sul da Bahia e Espírito Santo e uma percentagem bem maior (cerca de metade das espécies) de bromélias endêmicas para este mesmo trecho geográfico, foi encontrada nas matas úmidas de Una (Alves 2005). Embora comparações diretas entre as localidades devam ser tomadas com reserva, o padrão geral de diminuição da percentagem de espécies endêmicas ocorreu com bromélias epífitas de maneira semelhante ao ocorrido com a flora em geral. Enquanto as regiões de Una e Serra Grande apresentaram respectivamente 28,1 e $26,5 \%$ de endemismo de plantas vasculares (Amorim et al. 2005), a região de Jussari apresentou somente 7,3\% de espécies endêmicas para a região entre Bahia e Espírito Santo.

Uma vez que plantas epífitas são caracterizadas pelo alto endemismo (Koopowitz et al. 1993, Turner et al. 1994, Koopowitz 1994a, b) e pela preponderância da família Orchidaceae nas florestas tropicais da Ásia, África (Koopowitz et al. 1993, Tuner et al. 1994, Koopowitz 1994a, b) e Floresta Atlântica do Brasil (Kersten \& Silva 2001, Gonçalves \& Waechter 2002, Borgo \& Silva 2003, Giongo \& Waechter 2004), é provável que a percentagem de endemismo presente para as Bromeliaceae seja uma estimativa otimista do endemismo presente na família mais rica em espécies epífitas, no caso, Orchidaceae.

O número estimado de bromélias epífitas na RNST $(25 \pm 3,5)$ foi menor do que o número de bromélias epífitas estimado para a região de Una (33 $\pm 3,74$; Alves 2005) confirmando a afirmação de Gentry \& Dodson (1987) e Benzing (1990) de que áreas com baixa umidade ou com períodos de seca tendem a possuir um menor número de epífitas. Para a área investigada, o período de seca que ocorre nos meses de junho e julho deve ser um fator determinante para a diminuição do número de espécies epífitas. Hohenbergia disjuncta e H. blanchetiana são exemplos de espécies presentes na área e que ocorrem também em outras regiões do sul da Bahia com algum grau de perturbação e, portanto, sujeitas a algum grau de dessecação no dossel.

Várias características das Bromeliaceae e de epífitas indicam que o estabelecimento desta forma de vida nas árvores da RNST seja dificultado pela baixa retenção de umidade entre as árvores da floresta ocasionada pelas numerosas rochas no chão que distanciam as árvores umas das outras (Fontoura, obs. pess.). Embora as Bromeliaceae sejam capazes de ocupar locais extremamente xéricos (Benzing 2000) a presença de alguma fonte de umidade é necessária para que maiores densidades destas plantas possam ocorrer. Este é o caso de Nidularium procerum Lindman, $N$. innocentii Lemaire e de Aechmea bromeliifolia (Rudge) Baker que, embora terrestres, ocorrem em altas 
densidades (436/0,01 ha, 1364/0,03 ha e 150/0,015 ha respectivamente) somente em locais inundados da Floresta Atlântica (Freitas et al. 1998, Scarano et al. 2002). Estes trabalhos indicam que esta preferência pelos microhábitats inundados em comparação com os secos, ocorre como forma de aumentar a umidade do ar local, contribuindo para a melhor retenção de água nos tanques das bromélias (Scarano et al. 2002). A umidade retida nas cascas das árvores também é o principal fator para aumentar a abundância de Tillandsia usneoides (L.) L. nos troncos das árvores em florestas subtropicais dos Estados Unidos (Callaway et al. 2002). Uma vez que a região Jussari possui um período de seca de dois a três meses, é uma floresta semidecídua e os potenciais forófitos estão afastados entre si, parece improvável que a umidade existente na área seja suficiente para manter a riqueza e a abundância de epífitas em profusão.

A estabilização da curva de acumulação de espécies indica que a modificação na composição de espécies de bromélias é relativamente baixa dentro da faixa altitudinal analisada. Embora o primeiro jequitibá esteja a $285 \mathrm{~m}$ altitude e o último jequitibá se localize em uma altitude duas vezes maior (573 m), a abundância e o número de espécies epífitas é semelhante entre a maioria das árvores investigadas, entretanto, o único jequitibá com composição de espécies diferenciada, se localiza na maior altitude. Esta baixa modificação na composição de epífitas já foi assinalada por outros autores em localidades da América Central e em florestas de planície da Amazônia (Nieder et al. 1999, 2001), ocorrendo também na região de Una que possui altitude máxima de 100 m.s.m. (Alves 2005).

\section{Conclusão}

O registro de 19 morfoespécies e a estimativa de 25 espécies de bromélias epífitas a ocorrerem na RNST estão de acordo com o padrão de menor diversidade de epífitas em florestas mais secas. Embora haja baixa diversidade, a metodologia de acesso ao dossel foi fundamental para coletar epífitas que são exclusivas das copas das grandes árvores. Isto permitiu o registro de três espécies que não possuíam registro para a região nordeste da Floresta Atlântica e de um gênero ainda não coletado na área. Comparada a outras metodologias utilizadas para levantamentos florísticos, a metodologia empregada teve um baixo esforço amostral apresentando resultados semelhantes. As grandes árvores possuem especial importância para as epífitas devido à concentração de espécies e de indivíduos sobre as copas das árvores. Assim, seria aconselhável que outros levantamentos utilizassem metodologias de acesso ao dossel para coletar epífitas mais apropriadamente nas grandes árvores.

\section{Agradecimentos}

Este trabalho foi desenvolvido durante o III Curso de Campo de Ecologia de Dossel. As autoras são gratas aos proprietários da Reserva Particular do Patrimônio Natural da Serra do Teimoso, Henrique e Lucélia Berbert; à equipe de escaladores Iván Soler, Marcial Cotes, Sean Kerrigan, Márcia Rocca, Wesley da Duarte da Costa, Luiz Eduardo Azevedo Rocha, Tilson Nascimento; aos co-organizadores do curso Marcelo Mielke e Sérvio Ribeiro; aos financiadores e apoiadores do III Curso de Ecologia de Dossel: Universidade Estadual de Santa Cruz, Universidade Federal de Ouro Preto, Universidade Estadual de Campinas, Embaixada Britânica (FCO), Global Canopy Programme, Aliança da Mata Atlântica, Ministério do Meio Ambiente - Secretaria de Biodiversidade e Florestas, Instituto de Estudos Sócio Ambientais do Sul da Bahia (IESB). Por fim, as autoras são gratas ao curador do Herbário Cepec (A. Amorim) pelo exame da coleção de Bromeliaceae.

\section{Referências Bibliográficas}

ALVES, T.F. 2005. Distribuição geográfica, forófitos e espécies de bromélias epífitas nas matas e plantações de cacau da região de Una, Bahia. Tese de doutorado, Universidade Estadual de Campinas, Campinas.

AMORIM, A.M., FIASCHI, P., JARDIM, J.G., THOMAS, W.W., CLIFTON, B.C. \& CARVALHO, A.M. 2005. The vascular plants of a forest fragment in southern Bahia, Brazil. Sida, 21(3):1727-1752.

BENZING, D.H. 1990. Vascular epiphytes: general biology and related biota. Cambridge University Press, Cambridge.

BENZING, D.H. 2000. Bromeliaceae. Profile of an adaptive radiation. Cambridge University Press, Cambridge.

BORGO, M. \& SILVA, S.M. 2003. Epífitos vasculares em fragmentos de Floresta Ombrófila Mista, Curitiba, Paraná, Brasil. Revta. Bras. Bot. 26(3):391-401.

CALLAWAY, R.M., REINHART, K.O. \& MOORE, G.W. 2002. Epiphyte host preferences and host traits: mechanisms for species-specific interactions. Oecologia 132:221-230.

CARDELÚS, C.L., COLWELL, R.K. \& WATKINS Jr., J.E. 2006. Vascular epiphyte distribution patterns: explaining the mid-elevation richness peak. J. Ecol. 94:144-156.

COLWELL, R.K. \& CODINGTON, J.A. 1994. Estimating terrestrial biodiversity through extrapolation. Phil. Trans. R. Soc. Lond. B 345:101-118.

COLWELL, R.K. 1997. Estimates: statistical estimation of species richness and shared species from samples. Version 7.5. User's guide and application published at: http://viceroy.eeb.uconn.edu/estimates (último acesso em 10/01/2007)

FREITAS, C.A., SCARANO, F. R. \& WENDT, T. 1998. Habitat choice in two facultative epiphytes of the genus Nidularium (Bromeliaceae). Selbyana 19 (2): 236-239.

GENTRY, A. \& DODSON, C.H. 1987. Diversity and biogeography of Neotropical vascular epiphytes. Ann. Missouri Bot. Gard. 74:205-233.

GIONGO, C. \& WAECHTER, J. 2004. Composição florística e estrutura comunitária de epífitos vasculares em uma floresta de galeria na Depressão Central do Rio Grande do Sul. Revta. Bras. Bot. 27(3):563-572.

GONÇALVES, C.N. \& WAECHTER, J.L. 2002. Epífitos vasculares sobre espécimes de Ficus organensis isolados no norte da planície costeira do Rio Grande do Sul: padrões de abundância e distribuição. Acta Bot. Bras. 16(4):429-441.

GOTELLI, N. \& COLWELL, R.K. 2001. Quantifying biodiversity: procedures and pitfalls in the measurement and comparison of species richness. Ecol. Let. 4:379-391.

GOUVÊA, J.B.S., SILVA, L.A.M. \& HORI, M. 1976. Fitogeografia. In Diagnóstico sócio econômico da região cacaueira, (Comissão Executiva do Plano da Lavoura Cacaueira e Instituto Interamericano de Ciências Agrícolas, eds.). CEPLAC; IICA, Ilhéus, p.1-10.

GRADSTEIN, S.R., NADKARNI, N.M., KRÖMERT, T., HOLZ, I. \& NÖSKE, N. 2003. A protocol for rapid and representative sampling of vascular and non-vascular epiphyte diversity of tropical rain forests. Selbyana, 24(1):105-111.

JARDIM, J.G. 2003. Uma caracterização parcial da vegetação na região sul da Bahia, Brasil. In Corredor de Biodiversidade da Mata Atlântica do Sul da Bahia (P.I. Prado, E.C. Landau, R.T. Moura, L.P.S. Pinto, G.A.B. Fonseca \& K.Alger, orgs.). IESB; CI; CABS; UFMG; UNICAMP, Ilhéus. Publicação em CD-Rom.

KERSTEN, R.A. \& SILVA, S.M. 2001. Composição florística e estrutura do componente epifítico vascular em floresta da planície litorânea na Ilha do Mel, Paraná, Brasil. Revta. Bras. Bot. 24(2):213-226.

KOOPOWITZ, H., ANDERSEN, M., THORNHILL, A., NGUYEN, H. \& PHAM, A. 1994a. Comparison of distribution of terrestrial and epiphytic African orchids: implications for conservation. In Proceedings of the Fourteenth World Orchid Conference (A. Pridgeon, ed.). Her Majesty's Stationery Office, London, p. 120-124. 
KOOPOWITZ, H., THORNHILL, A. \& ANDERSEN, M. 1994b. A general stochastic model for prediction of biodiversity losses based on habitat conversion. Conserv. Biol. 8(2):425-438.

KOOPOWITZ, H., THORNHILL, A. \& ANDERSON, M. 1993. Species distribution profiles of the neotropical orchids Masdevallia and Dracula (Pleurothallidinae, Orchidaceae): implications for conservation. Biodiv. Cons. 2:681-690.

KRÖMER, T., KESSLER, M., GRADSTEIN, S.R. \& ACEBEY, A. 2005. Diversity patterns of vascular epiphytes along an elevational gradient in the Andes. J. Biogeogr. 32:1799-1809.

LANDAU, E.C. 2003. Padrões de ocupação espacial da paisagem da Mata Atlântica do sudeste da Bahia, Brasil. In Corredor de Biodiversidade da Mata Atlântica do Sul da Bahia (P.I. Prado, E.C. Landau, R.T. Moura, L.P.S. Pinto, G.A.B. Fonseca \& K. Alger, orgs.). IESB; CI; CABS; UFMG; UNICAMP, Ilhéus. Publicação em CD-Rom.

LEME, E.M. 1987. Novas Bromeliáceas do Brasil - V. Bradea, 4:392-405.

LEME, E.M. 1997. Canistrum: bromélias da Mata Atlântica. Salamandra, Rio de Janeiro.

LEME, E.M. 1999. New species of Brazilian Bromeliaceae: a tribute to Lyman B. Smith. Harv. Pap. Bot. 4(1):135-168.

MARTINI, A.M.Z., FIASCHI, P., AMORIM, A.M. \& PAIXÃO, J.L. 2007. A hot-point within a hot-spot: a high diversity site in Brazil's Atlantic Forest. Biodiv. Cons. 16:3111-3128.

MIGENIS, L.E. \& ACKERMAN, J.D. 1993. Orchid-phorophyte relationships in a forest watershed in Puerto Rico. J. Trop. Ecol. 9:231-240.

NIEDER, J., ENGWALD, S. \& BARTHLOTT, W. 1999. Patterns of neotropical diversity. Selbyana, 20(1):66-75.

NIEDER, J., PROSPERÍ, J. \& MICHALOUD, G. 2001. Epiphytes and their contribution to canopy diversity. Plant Ecol. 153:51-63.

PADGET, A. \& SMITH, B. 1989. On rope. National Speleological Society, Washington.

PERRY, D.R. 1978. A method of access into the crowns of emergent and canopy trees. Biotropica, 10(2):155-157.

SCARANO, F. R., DUARTE, H. M., RÔÇAS, G., BARRETO, S. M. B., AMADO, E., REINERT, F., WENDT , T., MANTOVANI, A., LIMA, H. R. P. \& BARROS, C. F. 2002. Acclimation or stress symptom? An integrated study of intraspecific variation in the clonal plant Aechmea bromeliifolia, a widespread CAM tank-bromeliad. Bot. J. Linnean Soc. 140: 391-401.

SEBBEN, A.M., KAGEYAMA, P.Y., SIQUEIRA, A.C.M.F. \& ZANATTO, A.C.S. 2000. Sistemas de cruzamento em populações de Cariniana legalis Mart. O. Ktze.: implicações para a conservação e o melhoramento genético. Scientia Florestalis 58:25-40.

SIQUEIRA-FILHO, J.A. \& LEME, E.M. 2006. Fragmentos de Mata Atlântica do nordeste: biodiversidade, conservação e suas bromélias. Andrea Jackobsson Estúdio Editorial Ltda., Rio de Janeiro.

SMITH, L.B. \& DOWNS, R.J. 1974. Bromeliaceae (Pitcairnioideae). Fl. Neotrop. 14(1):1-658.

SMITH, L.B. \& DOWNS, R.J. 1977. Bromeliaceae (Tillandsioideae). Fl. Neotrop. 14(2):663-1492.

SMITH, L.B. \& DOWNS, R.J. 1979. Bromeliaceae (Bromeliaceae). Fl. Neotrop. 14(3):1493-2142.

THE NEW YORK BOTANICAL GARDEN - NYBG. Neotropical Flora and Mycota Catalog http://www.nybg.org/bsci/hcol/netr/ (último acesso em 10/01/ 2006).

TURNER, I.M., TAN, H.T.W., WEE, Y.C., IBRAHIM, A.B., CHEW, P.T. \& CORLETT, R.T. 1994. A study of plant species extinction in Singapore: lessons for the conservation of tropical biodiversity. Conserv. Biol. 8(3):705-712.

UNIVERSIDADE ESTADUAL DE CAMPINAS - UNICAMP. NE 313 Tópicos em Ecologia de Campo II - Pesquisa em Dossel. http://www. ib.unicamp.br/profs/fsantos/ne313/index.html (último acesso em 10/01/2006).

WAECHTER, J.L. 1980. Estudo fitossociológico das orquidáceas da mata paludosa do Faxinal, Torres, Rio Grande do Sul. Dissertação de Mestrado, Universidade Federal do Rio Grande do Sul, Porto Alegre.

ZOTZ, G. \& VOLLRATH, B. 2003. The epiphyte vegetation of the palm Socratea exorrhiza - correlations with tree size, tree age and briophyte cover. J. Trop. Ecol. 19:81-90.

Recebido em 29/11/07 Versão reformulada recebida em 15/01/09 Publicado em 27/01/09 
